\title{
Heliospheric Transport of Neutron-Decay Protons
}

\author{
E. E. Chollet ${ }^{1}$ - R. A. Mewaldt ${ }^{1}$
}

(C) Springer $\bullet \bullet \bullet \bullet$

\begin{abstract}
We report on new simulations of the transport of energetic protons originating from the decay of energetic neutrons produced in solar flares. Because the neutrons are fast-moving but insensitive to the solar wind magnetic field, the decay protons are produced over a wide region of space, and they should be detectable by current instruments over a broad range of longitudes for many hours after a sufficiently large gamma-ray flare. Spacecraft closer to the Sun are expected to see orders-of-magnitude higher intensities than those at the Earth-Sun distance. The current solar cycle should present an excellent opportunity to observe neutron-decay protons with multiple spacecraft over different heliographic longitudes and distances from the Sun.
\end{abstract}

Keywords: Flares, Energetic Particles; Energetic Particles, Neutrons; Energetic Particles, Protons; Energetic Particles, Propagation

\section{Introduction}

Solar flares remain some of the best laboratories in the solar system for studying particle acceleration, production and interaction. The particles accelerated in flares either interact with the solar chromosphere, producing observable Xray and $\gamma$-ray emission, or escape into the interplanetary medium. In 1951, Biermann, Haxel and Schluter first suggested that solar flares could produce energetic neutrons, and evidence of these neutrons was subsequently observed in the 2.2-MeV neutron-capture $\gamma$-ray line, first by Chupp et al. (1973) and many times since. The first direct observations of solar-flare neutrons were made by Chupp et al. (1982). Detailed models of neutron production, transport, interaction and decay in flares have been developed (e.g. Lingenfelter et al., 1965, Hua and Lingenfelter, 1987, Murphy et al., 2007), allowing the number of interacting protons and escaping neutrons from a particular flare to be deduced from the $2.2 \mathrm{MeV}$ line fluence. The neutrons which escape the Sun undergo beta decay with a mean lifetime at rest of 886 seconds (about 14 minutes):

$$
n^{0} \longrightarrow p^{+}+e^{-}+\bar{\nu}_{e}
$$

\footnotetext{
1 California Institute of Technology, Mail Code 290-17, Pasadena, CA 91125 USA
} 
creating neutron-decay protons and electrons in the heliosphere.

The escaping neutrons and their decay products have been observed by both ground-based detectors and space-based instruments near the Earth and around the heliosphere (Chupp et al., 1982, Debrunner et al., 1983). Since the travel time between the Sun and Earth is $\geq 20$ minutes for particles with energies less than $100 \mathrm{MeV}$, most of the neutrons decay at some time during transit to $1 \mathrm{AU}$. The neutron-decay protons (Evenson, Meyer, and Pyle, 1983; Evenson et al., 1990) and also electrons (Dröge, Ruffolo, and Klecker, 1996) typically arrive before the original flare-accelerated protons (protons of the same energy accelerated in the flare site rather than originating from neutron decay), and before particles accelerated by the shock driven by the associated coronal mass ejection (CME), because they make part of their journey as neutrons that travel in a straight line and also do not lose energy.

Following the early work by Roelof (1966), several studies have investigated the effects that transport in the interplanetary magnetic field (IMF) can have on observations of neutron-decay protons, including their time-intensity profiles, energy spectra, and pitch-angle distributions (PADs). The first measurements of neutron-decay protons found the PADs were nearly isotropic, and, by fitting various aspects of the data, obtained scattering mean free-paths of $\lambda=0.3-0.5 \mathrm{AU}$ (Evenson, Meyer, and Pyle, 1983), $\lambda=0.2 \pm 0.005$ AU (Evenson et al., 1983), and $\lambda \sim 0.3 \mathrm{AU}$ (Evenson, Kroeger, and Meyer, 1985). In a more complete treatment Ruffolo (1991) modeled the transport of neutron-decay protons with 25 to $150 \mathrm{MeV}$ and fit the data from events summarized by Evenson, Kroeger, and Meyer| (1985). He considered pitch-angle scattering and adiabatic focusing of the protons and, by fitting "distance-traveled" distributions deduced from data obtained by the Third International Sun-Earth Explorer (ISEE-3), obtained the best-fit scattering mean free-paths for two events of $\lambda=0.26 \pm 0.05 \mathrm{AU}$ and $\lambda=0.37 \pm$ 0.06 AU. Recently, Agueda et al. (2011) modeled the transport of neutrons and their decay products to compare with observations of Solar Cycle 23 events.

Previous in situ studies of neutrons and their decay products were limited to a single vantage point, but new multi-point instrumentation is providing a different perspective on the geometry of energetic particles in the solar system. The canonical view (Reames, Kallenrode, and Stone, 1991; Nitta et al., 2006) holds that CME shocks distribute particles widely in longitude, and flare-associated protons and electrons cover a narrow $\left(\sim 40^{\circ}\right)$ range in longitude. However, recent studies have found that small flares can produce particles over $\sim 80^{\circ}$ latitude (Wibberenz and Cane, 2006, Wiedenbeck et al., 2009), while new imaging technology is routinely showing previously-undetectable CMEs associated with small flares. Understanding the longitude-distribution of neutron-decay protons may help unravel the diverse sources of energetic particles in the heliosphere. The twin spacecraft of the Solar TErrestrial RElations Observatory (STEREO, Kaiser et al. 2008) were launched in 2006 into orbits that lead (STEREOAhead) and trail (STEREO-Behind) the Earth. The Advanced Composition Explorer (ACE, Stone et al., 1998), Solar and Heliospheric Observatory (SOHO, Domingo et al. 1983), and Wind spacecraft (Acuna et al., 1995) are in orbit around Earth's L1 Lagrangian point, making multi-point studies covering more than $180^{\circ}$ in longitude possible in the current solar cycle. 


\section{Description of the Model}

To better understand what these spacecraft should observe in neutron-decay protons, we have created a model of neutron and proton transport in the heliosphere which determines the trajectories of a large number of individual particles by integrating their equations of motion. Neutrons are initially injected as a delta function in space and time at the solar surface, for simplicity set at $0^{\circ}$ latitude and longitude. The energy spectrum and angular distribution of the injected neutrons follow two of the examples that were investigated by Murphy et al. (2007), and illustrated in their Figure 15, where the flare-accelerated proton spectrum was assumed to be a power law with a spectral index of -4 , and the accelerated composition was characterizedby $\mathrm{He} / \mathrm{H}=0.5$ along with corresponding enrichments in the other species with atomic number $\mathrm{Z}>2$.

Murphy et al. (2007) varied two additional parameters: the mean free-path for pitch-angle scattering (PAS) of accelerated protons in the solar atmosphere (which we label as $\lambda_{s}$ to distinguish it from the scattering mean free-path in interplanetary space, which we label as $\lambda$ ) and the magnetic convergence parameter $(\delta)$. In Murphy et al. (2007) $\lambda_{s}$ is a dimensionless ratio of a) the distance for an arbitrary initial angular distribution to isotropize to b) the half-length of the loop on which the protons are trapped. They considered cases of no PAS $\left(\lambda_{s} \rightarrow \infty\right)$, intermediate PAS $\left(\lambda_{s}=300\right)$, and high scattering $\left(\lambda_{s}=20\right)$. The magnetic convergence parameter characterizes the magnetic field strength below the transition region, which is assumed to be proportional to the pressure to the power $\delta$. For $\delta=0$ there is no mirroring, and the angular distribution of the interacting protons is downward-isotropic. For $\delta=0.20$ the protons will mirror as the field increases with depth, leading to an angular distribution of escaping neutrons that is somewhat peaked at $90^{\circ}$ to the zenith, especially at energies $>10 \mathrm{MeV}$.

In this paper we consider two examples: Case 1 (where $\lambda_{s}=300$ ), and Case 2 (with $\lambda_{s} \rightarrow \infty$ ), both with $\delta=0.2$. The corresponding spectra of escaping neutrons are shown in Figure 15 of Murphy et al. (2007) in three angular ranges. We have approximated the escaping neutron spectra with piece-wise power laws covering three energy intervals and three angular ranges from $\cos \theta=0^{\circ}$ to $\cos \theta=90^{\circ}$ as specified in Tables 1 and 2. Here $\theta$ is the angle relative to the surface normal, with $\theta=0^{\circ}$ being the direction away from the Sun. We have considered neutrons with initial energies between 0.1 and $100 \mathrm{MeV}$, with flat spectra between 0.1 and $1 \mathrm{MeV}$ based on a projection of the curves in Figure 15 of Murphy et al. (2007). Because our code does not include relativistic corrections to the energy loss during transport, we do not consider particles with energies greater than $100 \mathrm{MeV}$.

Calculations based on observed 2.2-MeV line fluences have shown that in large flares between $\sim 3 \times 10^{28}$ and $\sim 10^{33}$ protons with energies $>30 \mathrm{MeV}$ are accelerated (Shih, Lin, and Smith, 2009), and the calculations of neutron production described above show that for the Case 1 spectra about 0.0054 escaping neutrons are produced for each accelerated $>30 \mathrm{MeV}$ proton (Murphy et al., 2007). For the Case 2 spectra where $\lambda_{s} \rightarrow \infty$ about 0.0073 escaping neutrons are produced for each accelerated proton $>30 \mathrm{MeV}$. The yields in Tables 1 and 2 are normalized to 1 flare-accelerated proton $>30 \mathrm{MeV}$. 
Table 1. Representation of the Escaping Neutron Spectra: Case $1^{1}$

\begin{tabular}{|c|c|c|c|c|c|c|c|}
\hline \multirow{2}{*}{$\begin{array}{l}\text { Energy } \\
(\mathrm{MeV})\end{array}$} & \multicolumn{2}{|c|}{$\cos \theta=0.0-0.3$} & \multicolumn{2}{|c|}{$\cos \theta=0.3-0.7$} & \multicolumn{2}{|c|}{$\cos \theta=0.7-1.0$} & \multirow{2}{*}{$\begin{array}{c}\cos \theta \geq 0 \\
\mathrm{~N} / \mathrm{P} 30^{3}\end{array}$} \\
\hline & Intercept $^{2}$ & Slope $^{2}$ & Intercept & Slope & Intercept & Slope & \\
\hline $0.1-1$ & $4.51 \mathrm{E}-5$ & 0.00 & $6.85 \mathrm{E}-5$ & 0.00 & $7.05 \mathrm{E}-5$ & 0.00 & $3.51 \mathrm{E}-4$ \\
\hline $1-10$ & $4.67 \mathrm{E}-5$ & -0.223 & $7.83 \mathrm{E}-5$ & -0.429 & $9.77 \mathrm{E}-5$ & -0.830 & $2.02 \mathrm{E}-3$ \\
\hline $10-100$ & $5.10 \mathrm{E}-4$ & -1.16 & $6.13 \mathrm{E}-4$ & -1.30 & $8.95 \mathrm{E}-4$ & -1.68 & $2.99 \mathrm{E}-3$ \\
\hline \multicolumn{8}{|l|}{$0.1-100$} \\
\hline $\mathrm{N} / \mathrm{P} 30^{3}$ & \multicolumn{2}{|c|}{$1.94 \mathrm{E}-3$} & \multicolumn{2}{|c|}{$2.38 \mathrm{E}-3$} & \multicolumn{2}{|c|}{$1.04 \mathrm{E}-3$} & $5.36 \mathrm{E}-3$ \\
\hline
\end{tabular}

${ }^{1}$ Case 1 is based on Figure 15b of (Murphy et al., 2007) with $\lambda_{s}=300$ and $\delta=0.20$ (see text) ${ }^{2}$ The Intercept and Slope entries define power-law representations of neutron energy spectra for the indicated energy and angular ranges.

${ }^{3} \mathrm{~N} / \mathrm{P} 30$ is the number of neutrons leaving the Sun for each accelerated proton $>30 \mathrm{MeV}$. N/P30 values in the last column and row are integrated over angle and energy respectively.

Table 2. Representation of the Escaping Neutron Spectra: Case $2^{1}$

\begin{tabular}{|c|c|c|c|c|c|c|c|}
\hline \multirow{2}{*}{$\begin{array}{c}\text { Energy } \\
(\mathrm{MeV})\end{array}$} & \multicolumn{2}{|c|}{$\cos \theta=0.0-0.3$} & \multicolumn{2}{|c|}{$\cos \theta=0.3-0.7$} & \multicolumn{2}{|c|}{$\cos \theta=0.7-1.0$} & \multirow{2}{*}{$\begin{array}{c}\cos \theta \geq 0 \\
\mathrm{~N} / \mathrm{P} 30^{3}\end{array}$} \\
\hline & Intercept $^{2}$ & Slope $^{2}$ & Intercept & Slope & Intercept & Slope & \\
\hline $0.1-1$ & $5.42 \mathrm{E}-5$ & 0.00 & $6.78 \mathrm{E}-5$ & 0.00 & $8.33 \mathrm{E}-5$ & 0.00 & $3.86 \mathrm{E}-4$ \\
\hline $1-10$ & $5.85 \mathrm{E}-5$ & -0.112 & $8.46 \mathrm{E}-5$ & -0.422 & $9.00 \mathrm{E}-5$ & -0.843 & $2.33 \mathrm{E}-3$ \\
\hline $10-100$ & $7.34 \mathrm{E}-4$ & -1.10 & $6.58 \mathrm{E}-4$ & -1.22 & $7.34 \mathrm{E}-4$ & -1.52 & $4.58 \mathrm{E}-3$ \\
\hline \multicolumn{8}{|l|}{$0.1-100$} \\
\hline $\mathrm{N} / \mathrm{P} 30^{3}$ & \multicolumn{2}{|c|}{$3.17 \mathrm{E}-3$} & \multicolumn{2}{|c|}{$2.95 \mathrm{E}-3$} & \multicolumn{2}{|c|}{$1.17 \mathrm{E}-3$} & $7.29 \mathrm{E}-3$ \\
\hline
\end{tabular}

${ }^{1}$ Case 2 is based on Figure 15a of (Murphy et al., 2007) with $\lambda_{s} \rightarrow \infty$ and $\delta=0.20$ (see text)

${ }^{2}$ The Intercept and Slope entries define power-law representations of neutron energy spectra for the indicated energy and angular ranges.

${ }^{3} \mathrm{~N} / \mathrm{P} 30$ is the number of neutrons leaving the Sun for each accelerated proton $>30 \mathrm{MeV}$.

$\mathrm{N} / \mathrm{P} 30$ values in the last column and row are integrated over angle and energy respectively.

At each timestep in the simulation, the escaping neutrons are tested for decay by comparing a random number between 0 and 1 with a decay probability given by $P_{d}=\Delta t / \tau_{d}$ where $\tau_{d}$ is the mean lifetime (corrected for time dilation). Once a neutron decays, the trajectory of the resulting proton is followed as it begins to feel the effects of the interplanetary magnetic field, assumed to be a Parker spiral for a solar wind speed of $400 \mathrm{~km} \mathrm{~s}^{-1}$. Rather than computing the gyromotion directly, we treat the particles adiabatically, that is, we assume the perpendicular kinetic energy over the magnetic field magnitude $\left(W_{\perp} / B\right)$ is constant in the frame corotating with the Sun. This scheme produces results in excellent agreement with schemes where the gyromotion is integrated directly, but results in a significant savings of computational resources. The proton trajectory is followed until it is lost to the Sun, passes the outer boundary at 10 astronomical 
units (AU, $1 \mathrm{AU}$ is the Earth-Sun distance), or until it has been in transit for 10 days.

Our code includes the effects of pitch-angle scattering and adiabatic cooling of the protons in the expanding solar wind. The particle scattering is included in a manner similar to the particle decay, where a scatter probability is compared to a random number for each timestep. The form of the scattering mean free-path is derived from the quasilinear theory (Jokipii, 1966; Giacalone and Jokipii, 1999):

$$
\lambda_{Q L T}(r, v)=0.1\left(\frac{v}{v_{o}}\right)^{\frac{1}{3}}\left(\frac{B(1 A U)}{B(r)}\right)^{\frac{1}{3}},
$$

where $r$ is radial distance, $v$ is particle velocity, $B$ is the strength of the IMF, and $v_{o}$ is the velocity of a $1-\mathrm{MeV}$ proton.

Our value for the scattering mean free-path $\lambda$ ranges from 0.17 to $0.22 \mathrm{AU}$ for $25-150 \mathrm{MeV}$ protons at $1 \mathrm{AU}$ and gradually decreases closer to the Sun, values that agree well with the previously observed PADs in neutron-decay proton events. The recent study of neutron-decay protons by Agueda et al. (2011) assumed scatter-free propagation, in which the decay-protons quickly focus into a beam aligned with the IMF. In such a beam, protons cross a detector at 1 AU only once. In our simulation, $10-\mathrm{MeV}$ protons cross $1 \mathrm{AU}$ an average of 6-7 times.

In an earlier paper reporting on simulations of solar energetic particle (SEP) transport, Chollet, Giacalone, and Mewaldt (2010) found that the proton energy deposition at $1 \mathrm{AU}$ was constant to within a factor of two as the scattering mean free-path was varied from $\lambda=0.01$ to $\lambda=1 \mathrm{AU}$. It was also relatively insensitive to variations in the radial dependence of $\lambda$. This is because when pitch-angle scattering is increased the protons cross $1 \mathrm{AU}$ more times on average, but they also lose more energy (see Figure 7 in Chollet, Giacalone, and Mewaldt (2010)). If $\lambda$ is increased, we expect about the same proton fluence, but distributed over a shorter time period, with higher average intensity. As a result, we believe the results of our simulations are relatively insensitive to the details of our description of pitch-angle scattering. The events observed to date suggest that the inclusion of some scattering scheme is necessary if the simulation is to match the details of real events.

Our simulation outputs the times particles cross a sphere at a given distance from the Sun along with their positions and energies, allowing intensities and fluences to be calculated at the simulated spacecraft. Calculations based on observed 2.2-MeV line fluences have shown that in large flares between $\sim 3 \times 10^{28}$ and $\sim 10^{33}$ protons with energies $>30 \mathrm{MeV}$ are accelerated (Shih, Lin, and Smith, 2009).

The calculations of neutron production described above show that for the Case 1 spectra about 0.0054 escaping neutrons are produced for each accelerated $>30 \mathrm{MeV}$ proton (Murphy et al., 2007). For the Case 2 spectra where $\lambda_{s} \rightarrow \infty$ about 0.0073 escaping neutrons are produced for accelerated proton $>30 \mathrm{MeV}$. Therefore, we have normalized the intensities and fluences to the Case 1 and Case 2 yields of $5.36 \times 10^{29}$ and $7.29 \times 10^{29}$ injected neutrons expected for $10^{32}$ accelerated protons $>30 \mathrm{MeV}$, somewhat greater than the median value observed in real flares. [At least $23 \%$ of the Ramaty High Energy Solar Spectroscopic 


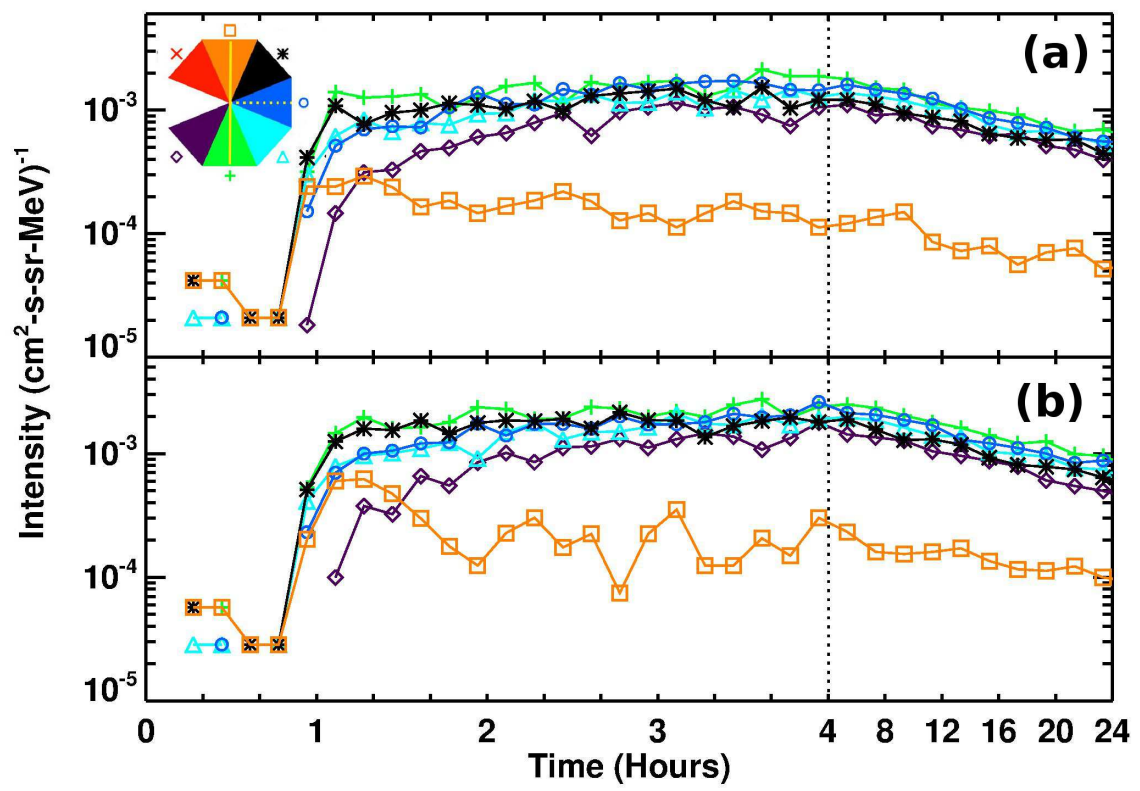

Figure 1. (a) Intensities of 1 to $10 \mathrm{MeV}$ protons at $1 \mathrm{AU}$ for spacecraft at different longitudes following a central-meridian $0^{\circ}$-latitude $\gamma$-ray flare with $10^{32}$ accelerated protons $>30 \mathrm{MeV}$ and Case- 1 characteristics $\left(\lambda_{s}=300\right.$ and $\delta=0.20$; see Murphy et al. (2007)). The time T $=0$ marks the time the flare photons would be observed at Earth. To the left of the black dotted line, the intensities are binned in 10-minute increments, and to the right of the dotted line the intensities are binned in 2-hour increments. For all but the orange curve the mean statistical uncertainty is $\sim 18 \%$ for the 10 -minute points and $\sim 8 \%$ for the 2-hour points. For the orange curve the mean statistical error is $\sim 43 \%$ for the 10 -minute points and $\sim 19 \%$ for the 2-hour points. The different colors and symbols represent the different virtual spacecraft, with a legend at the top left indicating the relative longitudes of the spacecraft. The dotted line in the legend is the radial direction from the injection point. The sector represented by the red $\mathrm{x}$ has intensities a factor of 10 lower than the adjacent sector, and so is off the scale in this plot. (b) Same as panel (a) but for Case 2 neutron spectra characterized by $\lambda_{s} \rightarrow \infty$ and $\delta=0.20$. The mean statistical uncertainties are similar to those in panel (a). Both simulations used the same sequence of random numbers.

Imager (RHESSI) $\gamma$-ray flares in which the $2.2 \mathrm{MeV}$ line was detected were this large (Shih, Lin, and Smith, 2009); in some events RHESSI missed some of the emission because of shadowing by Earth.] Our total Case 2 yield of 1-100 MeV escaping neutrons is about a factor of $\sim 3.5$ greater than for similar neutron spectra in Hua et al. (2002), which was the basis of the transport study by Agueda et al. (2011). This is likely because Hua et al. assumed an accelerated composition less rich in He and heavier ions, as well as a harder proton spectrum (spectral index of -3.5).

\section{Results and Discussion}

Figure 1 shows Case 1 and Case 2 intensities of energetic protons vs. time for a simulated spacecraft at 1 AU located at different heliographic longitudes. Each 
of the seven simulated spacecraft integrate over $45^{\circ}$ in longitude and $15^{\circ}$ in latitude. The 1-AU intensities reflect the greater yield of escaping neutrons in Case 2, in most directions the intensities are somewhat higher in Figure 1b than in Figure 1a. Increasing the amount of PAS decreases the yield of escaping neutrons because more accelerated protons are scattered into the loss cone to the chromosphere, so that they interact deeper in the atmosphere while typically headed downward. Since the secondary neutrons generally go forward at high energy, fewer neutrons escape. When PAS is ignored, more neutrons interact while mirroring and so there is increased neutron escape near the solar horizon $\left(\theta=90^{\circ}\right)$.

The intensities in Figure 1 are above the background levels of some currentlyavailable instruments over more than half of the longitudinal circle for most of a day. The Low Energy Telescope (LET) and High Energy Telescope (HET) sensors on STEREO, which observe protons between 2 and $100 \mathrm{MeV}$, have a galactic cosmic ray background level of a few times $10^{-5}\left(\mathrm{~cm}^{2} \text {-s-sr-MeV }\right)^{-1}$ at solar maximum (see also Gómez-Herrero et al., 2010, Leske et al., 2008, and Mewaldt et al., 2009). With this instrument background level, the simulated event would be detectable at five of the virtual spacecraft in this simulation $\left(225^{\circ}\right)$ for a few hours to a day. The estimated decay-proton intensities for the 28 October 2003 "Halloween" event would be $\sim 10$ times greater. Agueda et al. (2011) conducted a survey of 1 to $11 \mathrm{MeV}$ Wind-3DP data for eight X-class flares, and found no evidence for detection of neutron-decay protons. However, their background levels were $\sim 0.04-0.1 \mathrm{~cm}^{-2} \mathrm{sr}^{-1} \mathrm{MeV}^{-1}$ for 3-6 MeV protons and $\sim 0.02 \mathrm{~cm}^{-2} \mathrm{sr}^{-1} \mathrm{MeV}^{-1}$ for $6-11 \mathrm{MeV}$ protons, and they note that their simulated neutron-decay proton spectrum is well below the observed background proton spectrum for the only one of these events in which RHESSI observed $2.2-\mathrm{MeV} \gamma$-ray emission.

Though the intensities predicted by our simulation are above instrument detection thresholds, for magnetically well-connected events it is difficult to distinguish neutron-decay protons from shock and flare accelerated protons (Roelof, 1966). Events originating in the eastern hemisphere are more likely to be detected because the directly-accelerated protons often arrive several hours after the neutron-decay products. However, many of the largest SEP events are preceded by earlier events that elevate interplanetary SEP levels, which may explain why so few detections of neutron-decay events have been reported.

In Figure 2 we show snapshots of particle positions in the ecliptic plane 20 minutes and one hour after the flare, with the angles and symbols of the virtual spacecraft superimposed. This simulation shows that solar flares provide a means of quickly distributing neutron-decay protons (and electrons) over more than $180^{\circ}$ in longitude within the first hour following the flare. The solar surface creates a solid horizon that neutrons cannot cross. If the neutron-decay protons travel without scattering along interplanetary magnetic field lines, this horizon will create a shadowed region in interplanetary space as discussed by Evenson, Meyer, and Pyle (1983) and Agueda et al. (2011). The scattering included in this simulation allows protons to move against the solar wind flow into this shadowed region, albeit with difficulty. As a result, a spacecraft like 
20 minutes

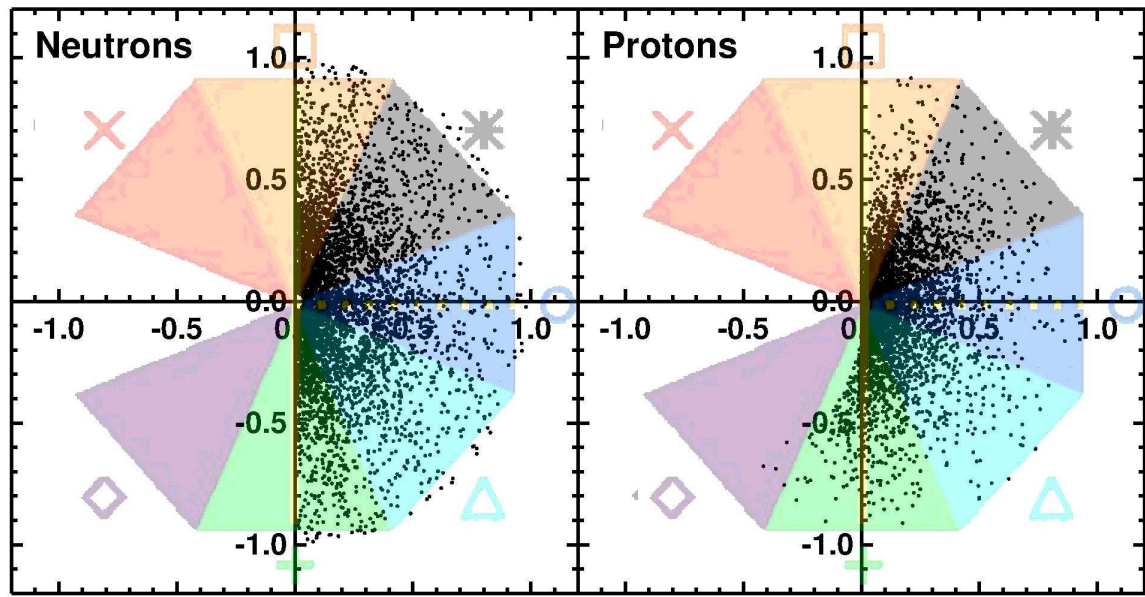

60 minutes

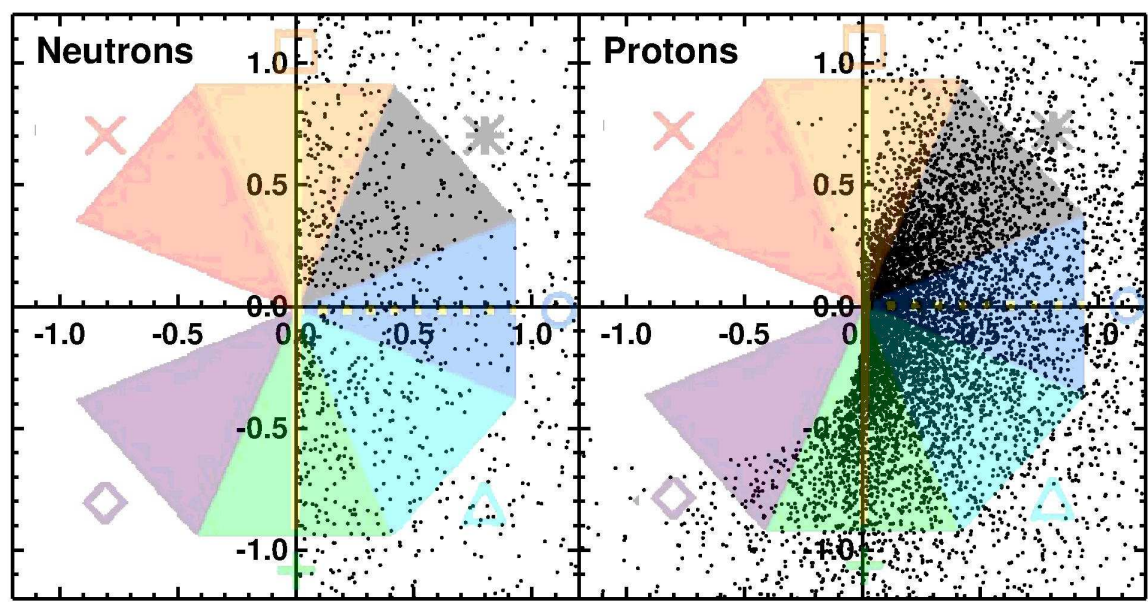

Figure 2. Snapshots of particle positions at 20 and 60 minutes after the neutrons are injected (in 20 minutes a $100 \mathrm{MeV}$ neutron travels just over $1 \mathrm{AU}$ ). The left half shows the positions of the neutrons, while the right half shows the positions of neutron-decay protons. A legend representing the sectors of the virtual spacecraft is superimposed. The neutrons travel in straight lines, while the protons spiral while following the magnetic field. This simulation used the Case-1 neutron spectra that includes PAS of the accelerated protons with $\lambda_{s}=300$ and $\delta$ $=0.20$ (see text and Murphy et al. (2007)) .

Messenger that happens to be inside the shadow might still be able to detect neutron-decay protons that diffuse into the shadow region.

Murphy et al. (2007) found that more neutrons are produced close to the neutron horizon than in the radial direction (see Tables 1 and 2, where the minimum neutron intensities are roughly normal to the flare site), and the interplanetary results reflect this. Under the influence of the interplanetary magnetic field neutron-decay protons move clockwise from the sector marked with a square 


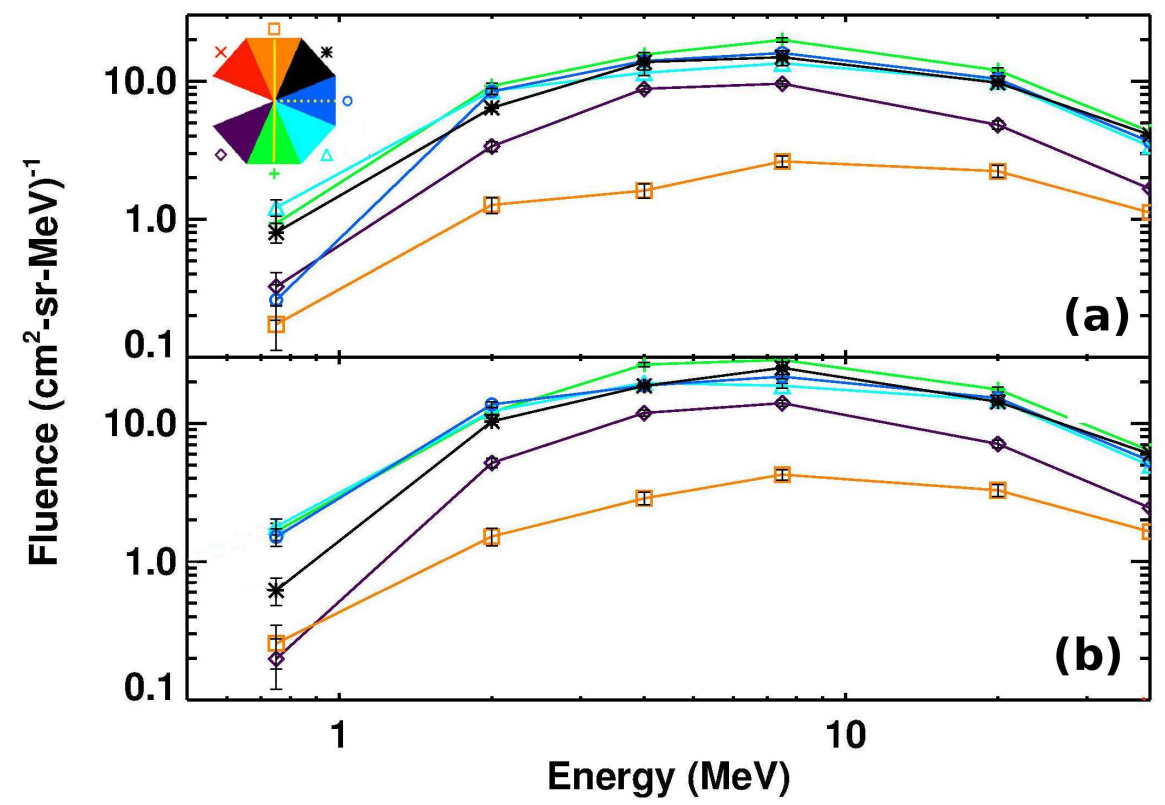

Figure 3. Proton fluence spectra integrated over the first four hours following neutron release (assumed to be the peak of the flare) are shown for each of the virtual spacecraft following a central-meridian $\gamma$-ray flare with $10^{32}$ accelerated protons $>30 \mathrm{MeV}$. The relative intensities at the different spacecraft reflect the time-intensity profiles in Figure 1. Low-energy protons below $0.5 \mathrm{MeV}$ have not yet reached $1 \mathrm{AU}$. Panel (a) is based on Case 1 neutron spectra with $\lambda_{s}=300$ and $\delta=0.20$. Panel (b) is based on Case 2 neutron spectra with $\lambda_{s} \rightarrow \infty$ and $\delta=$ 0.20 .

$\left(\mathrm{W} 67.5^{\circ}-\mathrm{W} 112.5^{\circ}\right)$ to the sector marked with an asterisk $\left(\mathrm{W} 22.5^{\circ}-\mathrm{W} 67.5^{\circ}\right)$, and from one half of the sector marked with a plus $\left(\mathrm{W} 45^{\circ}-\mathrm{W} 67.5^{\circ}\right)$ to the other $\left(\mathrm{W} 22.5^{\circ}-\mathrm{W} 45^{\circ}\right)$, making the plus and asterisk sectors the highest intensities early in the event (see also Figure 1). Later in the event, the particles have moved even more clockwise into the diamond (E112.5 $\left.{ }^{\circ} \mathrm{E} 157.5^{\circ}\right)$ and circle $\left(\mathrm{E} 22.5^{\circ}-\right.$ $\left.\mathrm{W} 22.5^{\circ}\right)$ sectors, while the square $\left(\mathrm{W} 67.5^{\circ}-\mathrm{W} 112.5^{\circ}\right)$ sector quickly becomes depleted.

Proton fluence spectra at 1 AU integrated over the first four hours following the neutron injection are presented for each sector in Figure 3. Most of the lowspeed protons (with energies less than a few $\mathrm{MeV}$ ) have not yet reached $1 \mathrm{AU}$ at this time. The higher-speed protons reflect the injection spectra and transport effects. As mentioned above, Agueda et al. (2011) have simulated neutron-decay proton intensities at 1-AU using somewhat different neutron spectra and interplanetary transport conditions. Their intensity spectra are similar in shape to our fluence spectra (Figure 3), but our spectra show evidence for adiabatic energy loss below $\sim 10 \mathrm{MeV}$. In addition, their predicted peak intensities are greater than ours, presumably because, without pitch-angle scattering, all of the protons of a given energy arrive within a narrow time interval. 


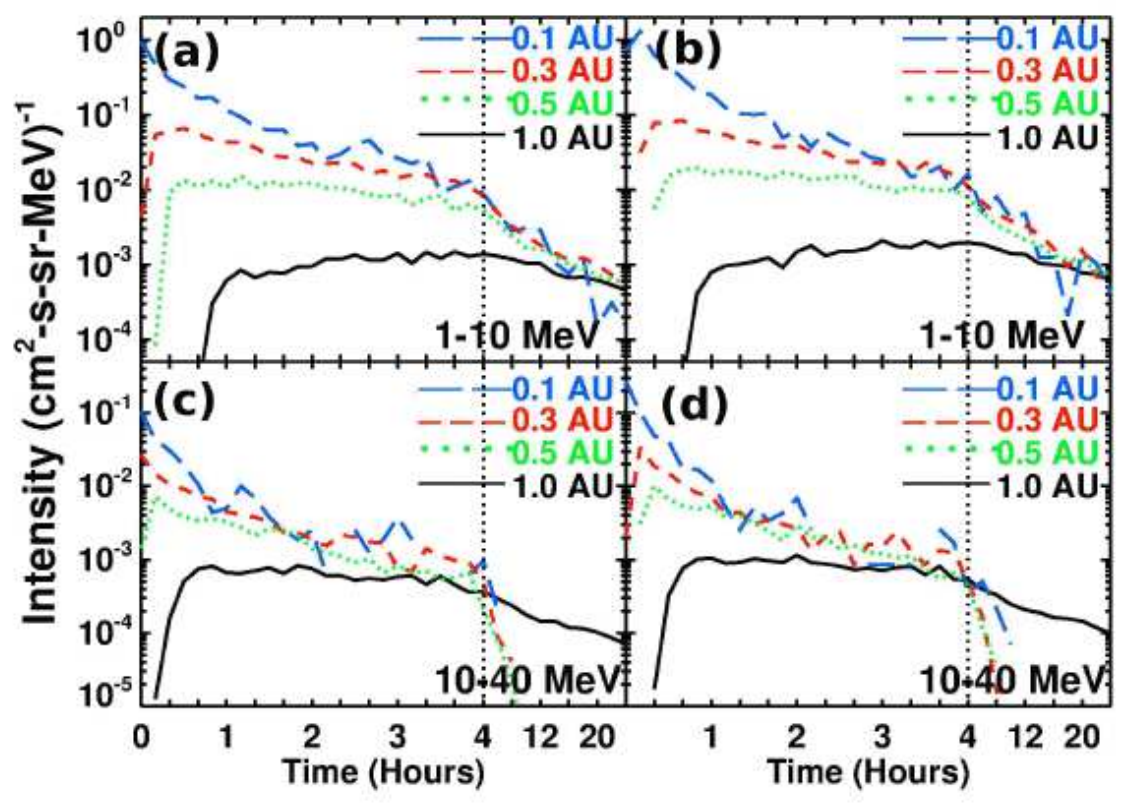

Figure 4. Proton intensities are shown for spacecraft at $0.1,0.3,0.5$ and $1 \mathrm{AU}$ following a central-meridian $\gamma$-ray flare with $10^{32}$ accelerated protons $>30 \mathrm{MeV}$. Panels (a) and (c) are based on Case 1 neutron spectra while Panel (b) and (d) are based on Case 2 neutron spectra. To the left of the black dotted line, the intensities are binned in 10-minute increments, and to the right of the dotted line the intensities are binned in 2-hour increments. The virtual spacecraft in this case are in the magnetically-well-connected sector spanning $45^{\circ}$, so the black solid lines in this plot are the same as the cyan triangle lines in Figure 1. The mean statistical error on the 10-minute points increases closer to the Sun, and is $\sim 10 \%$ on the 0.5 AU line, $\sim 20 \%$ on the 0.3 AU line and $\sim 40 \%$ on the 0.1 AU line.

As expected, these simulation results show that neutron-decay protons should be more easily detectable at heliocentric distances less than 1 AU. The simulated spacecraft in Figure 4 are all in the magnetically-connected sector $\left(45^{\circ}\right.$ clockwise from radial) but at different distances from the Sun, so the 1 AU results in the top portion of Figure 4 are the same as the results for that sector in Figure 1. Within 0.5 AU heliocentric distance, neutron-decay protons peak within the first hour, and remain well above nominal background levels for a full day. The peak fluxes at $0.1 \mathrm{AU}, 0.3 \mathrm{AU}$ and $0.5 \mathrm{AU}$ are respectively $\sim 1000, \sim 100$ and $\sim 10$ times higher than at 1 AU. The Solar Orbiter and Solar Probe Plus missions, which will approach to within 62 and 9.5 solar radii of the Sun (respectively), will carry instrumentation that should detect neutron-decay protons and electrons in a number of events.

Close to the Sun, a shock from the associated CME can overtake neutrondecay protons with energies of a few $\mathrm{MeV}$ or less if their radial transport is stalled by pitch-angle scattering (see Figure 2). Thus neutron-decay protons may provide seed particles for acceleration by CME-driven shocks, as suggested by Feldman et al. (2010). 


\section{Conclusions}

The current solar cycle should present an excellent opportunity to observe neutron-decay protons with multiple spacecraft over different heliographic longitudes and distances from the Sun. Our simulation suggests that these neutrondecay protons are very rapidly distributed over more than $180^{\circ}$ in longitude and should be detectable by current instrumentation at more than one longitude following large $\gamma$-ray events, provided the pre-event energetic particle background is sufficiently quiet. Spacecraft closer to the Sun such as MESSENGER, and eventually Solar Probe Plus and Solar Orbiter, will observe even higher intensities of neutron-decay protons. While the decay protons and electrons with energies greater than a few $\mathrm{MeV}$ quickly escape into the heliosphere, lower-energy protons linger close to the Sun, where they are available to be accelerated by a CME shock into high-energy particles.

Acknowledgements This work was supported by NASA under grants NNX08AI11G and NNX11AO75G, and subcontract SA2715-26309 from UC Berkeley under NASA contract NAS5-03131. We appreciate the assistance of R. A. Leske and C. M. S. Cohen, and discussions with R. Murphy. We also thank the anonymous reviewer for a number of helpful comments and suggestions.

\section{References}

Acuna, M.H., Ogilvie, K.W., Baker, D.N., Curtis, S.A., Fairfield, D.H., and Mish, W.H.: 1995, The Global Geospace Science Program and its Investigations, Space Sci. Rev. 71, 5-21.

Agueda, N., Krucker, S., Lin, R.P., and Wang, L.: 2011, On the Near-Earth Observation of Protons and Electrons from the Decay of Low-Energy Solar Flare Neutrons, Astrophys. J. 737, 1-7.

Biermann, Von L., Haxel, O., and Schlŭter, A.: 1951, Neutrale Ultrastrahlung von der Sonne, Zs. Naturforsch. 6a, 47-48.

Chollet, E.E., Giacalone, J., and Mewaldt, R.A.: 2010, Effects of Interplanetary Transport on Derived Energetic Particle Source Strengths, J. Geophys. Res., 115, A06101, doi:10.1029/2009JA014877.

Chupp, E.L., Forrest, D.J., Higbie, P.R., Suri, A.N., Tsai, C., and Dunphy P.P.: 1973, Solar Gamma Ray Lines observed during the Solar Activity of August 2 to August 11, 1972, Nature 241, 333-335.

Chupp, E.L., Forrest, D.J., Ryan, J.M., Heslin, J., Reppin, C., Pinkau, K., et al.: 1982, A Direct Observation of Solar Neutrons Following the 0118 UT Flare on 1980 June 21, Astrophys. J 263, L95-L99.

Debrunner, H., Flueckiger, E., Chupp, E.L., and Forrest, D.J.: 1983, The solar cosmic ray neutron event on June 3, 1982, Proc. Int. Cosmic Ray Conf. 4, 75-78.

Domingo, V., Fleck, B., and Poland, A.: 1995, The SOHO Mission: An Overview, Solar Physics 162, $1-37$.

Dröge, W., Ruffolo, D., and Klecker, B.: 1996, Observations of Electrons from the Decay of Solar Flare Neutrons, Astrophys. J. 464, L87-L90.

Evenson, P., Meyer, P., and Pyle, K.R.: 1983, Protons from the Decay of Solar Flare Neutrons, Astrophys. J. 274, 875-882.

Evenson, P., Kroeger, R., Meyer, P., and Muller, D.: 1983, Solar Flare Neutron Fluxes Derived from Interplanetary Charged Particle Measurements, Proc. 18th Internat. Cosmic Ray Conf. 4, 97-100.

Evenson, P., Kroeger, R., and Meyer, P.: 1985, Further Observations of Protons Resulting from the Decay of Neutrons Ejected by Solar Flares, Proc. 19th Internat. Cosmic Ray Conf. 4, $130-133$. 
Evenson, P., Kroeger, R., Meyer, P., and Reames, D.: 1990, Solar Neutron Decay Proton Observations from Cycle 21, Astrophys. J. Supp. 73, 273-277.

Feldman, W.C., Lawrence, D.J., Goldsten, J.O., Gold, R.E., Baker, D.N., Haggerty, D.K., et al.: 2010, Evidence for Extended Acceleration of Solar-Flare Ions from 1-8 MeV Solar Neutrons Detected with the MESSENGER Neutron Spectrometer, J. Geophys. Res. 115, A01102, doi:10.1029/2009JA014535.

Giacalone, J. and Jokipii, J.R.: 1999, The Transport of Cosmic Rays Across a Turbulent Magnetic Field, Astrophys. J. 520, 204-214.

Gómez-Herrero, R., Malandraki, O., Dresing, N., Kilpua, E., Heber, B., Klassen, A., et al.: 2010, Multi-point Observations of CIR-Associated Energetic Particles during 2008 Solar Minimum, In: Maksimovic, M., Issautier, K., Meyer-Vernet, N., Moncuquet, M., and Pantellini, F. (eds), 12th International Solar Wind Conference, AIP Conference Proceedings 1216, American Institute of Physics, 608-612.

Hua, X.-M., Koslovsky, B., Lingenfelter, R.E., Ramaty, R., and Stupp A.: 2002, Angular and Energy-Dependent Neutron Emission from Solar Flare Magnetic Loops, Astrophys. J. Suppl. 140, 563-579.

Hua, X.-M. and Lingenfelter, R.E.: 1987, Solar Flare Neutron and Accelerated Ion Angular Distributions, Astrophys. J. 323, 779-794.

Jokipii, J.R.: 1966, Cosmic-Ray Propagation, I. Charged Particles in a Random Magnetic Field, Astrophys. J. 146, 480-487.

Kaiser, M.L., Kucera, T.A., Davila, J.M., St. Cyr, O.C., Guhathakurta, M., and Christian, E.: 2008, The STEREO Mission: An Introduction, Space Sci. Rev. 136, 5-16.

Leske, R.A., Mewaldt, R.A., Mason, G.M., Cohen, C.M.S., Cummings, A.C., Davis, A.D., et al.: 2008, STEREO and ACE Observations of CIR Particles, In: Li, G., Hu, Q., Verkhoglyadova, O., Zank, G.P., Lin, R.P., and Luhmann, J. (eds), Particle Acceleration and Transport in the Heliosphere and Beyond, AIP Conference Proceedings 1039, American Institute of Physics, 131-136.

Lingenfelter, R.E., Flamm, E.J., Canfield, E.H., and Kellman, S.: 1965, High-Energy Solar Neutrons, 1, Production in Flares, J. Geophys. Res. 70, 4077-4086.

Mewaldt, R.A., Leske, R.A., Stone, E.C., Barghouty, A.F., Labrador, A.W., Cohen, C.M.S., et al.: 2009, STEREO Observations of Energetic Neutral Hydrogen Atoms during the 5 December 2006 Solar Flare, Astrophys. J. 693, L11-L15.

Murphy, R.J., Kozlovsky, B., Share, G.H., Hua, X.-M., and Lingenfelter, R.E.: 2007, Using Gamma-Ray and Neutron Emission to Determine Solar Flare Accelerated Particle Spectra and Composition and the Conditions Within the Flare Magnetic Loop, Astrophys. J. 168, $167-194$

Nitta, N.J., Reames, D.V., De Rosa, M.L., Liu, Y., Yashiro, S., and Gopalswamy, N.: 2006, Solar Sources of Impulsive Solar Energetic Particle Events and Their Magnetic Field Connection to the Earth, Astrophys. J. 650, 438-450.

Reames, D.V., Kallenrode, M.-B., and Stone, R.G.: 1991, Multispacecraft Observations of Solar ${ }^{3}$ He-Rich Events, Astrophys. J. 380, 287-292.

Roelof, E.C.: 1966, Effect of the Interplanetary Magnetic Field on Solar Neutron-Decay Protons, J. Geophys. Res. 71, 1305-1317.

Ruffolo, D.: 1991, Interplanetary Transport of Decay Protons from Solar Flare Neutrons, Astrophys. J. 382, 688-698.

Shih, A.Y., Lin, R.P., and Smith, D.M.: 2009, RHESSI Observations of the Proportional Acceleration of Relativistic $>0.3 \mathrm{MeV}$ Electrons and $>30 \mathrm{MeV}$ Protons in Solar Flares, Astrophys. J. 698, L152-L157.

Stone, E.C., Frandsen, A.M., Mewaldt, R.A., Christian, E.R., Margolies, D., Ormes, J.F., and Snow, F.: 1998, The Advanced Composition Explorer, Space Sci. Rev. 86, 1-24.

Wiedenbeck, M.E., Mason, G.M., Gómez-Herrero, R., Haggerty, D.K., Nitta, N.V., Cohen, C.M.S., et al.: 2009, Observations of a ${ }^{3}$ He-rich SEP Event over a Broad Range of Heliographic Longitudes: Results from STEREO and ACE, In: Maksimovic, M., Issautier, K., Meyer-Vernet, N., Moncuquet, M., and Pantellini, F. (eds), 12th International Solar Wind Conference, AIP Conference Proceedings 1216, American Institute of Physics, 621-624.

Wibberenz, G. and Cane, H.V.: 2006, Multi-Spacecraft Observations of Solar Flare Particles in the Inner Heliosphere, Astrophys. J. 650, 1199-1207. 